\title{
Automatic Alarm of Tobacco Blending Accuracy Based on PLC Control System
}

\author{
Yanyan $\mathrm{CaO}^{\mathrm{a}}$, Ligong Cui ${ }^{\mathrm{b}}$, Qian $\mathrm{Lv}^{\mathrm{c}}$ \\ Department of electrical engineering, Binzhou Polytechnic, Binzhou, Shandong, China \\ e-mail: ayaya_sd@163.com, 'blg_cui@bzpt.edu.cn, 'cqianqiandexiao@qq.com
}

\begin{abstract}
The tobacco blending is a special process in the tobacco product line. It plays a very important role in this area because it will affect the quality of the tobacco. The instant blending accuracy of the technological requirement is higher than $85 \%$. This paper introduces an automatic alarm of tobacco blending accuracy based on PLC control system. The automatic alarming system is available and we can greatly improve the producing and automatic level. This is worth extending in the tobacco industry.
\end{abstract}

Keywords: Blending accuracy, Automatic alarm, PLC control

\section{Introduction}

The 5000kg tobacco product line in Qingzhou Cigarette Factory is under the model of German rainbow, which is designed and produced by Kunchuan Company. The bottom electronic control system is the PLC of Siemens S400, and the monitoring system is CITECT through computer. The intermediate connection is Ethernet cable and Repeaters.

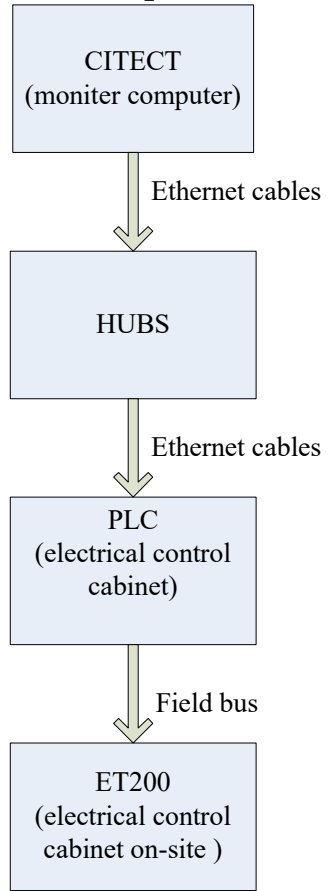

Fig.1 Framework of electronic control system 
The tobacco blending scale is designed by the Jiujiang 707 research institution. The bottom electronic control system is the PLC of Siemens S300, man-machine interaction with Siemens touch screen and INTOUCH monitoring software. The intermediate control and the while line is connected by profibus and Ethernet.

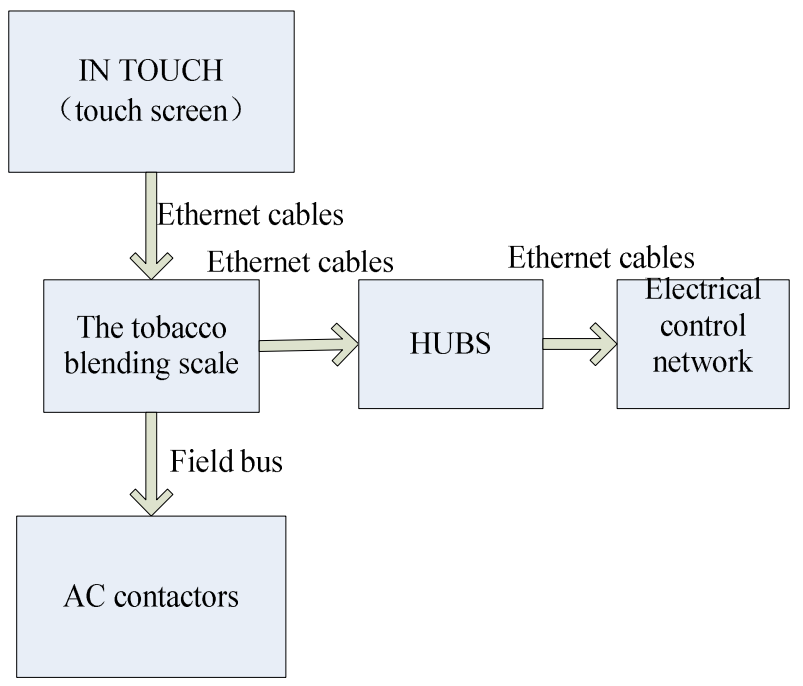

Fig. 2 Framework of electronic control system in the tobacco blending

The tobacco blending is a special process in the tobacco product line. Those five slices, which are leaf slice, peduncle slice, bulking slice, man-made slice and recycled slice, are blended with certain proportion under strict technology standard. After this, these slices will become semi-finished products for further fragrance adding. The blending precision will directly affect the quality of the tobacco. The instant blending accuracy of the technological requirement must be higher than $85 \%$. However, this process is only monitored by operators on the scene. Problems cannot be found immediately, which influences the blending accuracy and thus lowers the tobacco quality.

\section{Cause analysis}

Here we have an investigation statistics show the reason why the accuracy is lowered during the instant blending.

Here are two main reasons

Machinery

Chain broken:

The control machine is under operation but the belt does not work. Tobacco slices cannot be blended and the precision is low.

Reduction box broken:

The control machine is under operation but the reduction box does not, which leads to the stillness of the transmission belt. Tobacco slices cannot be blended and the precision is low.

Transmission belt broken:

The transmission belt slips or adheres, the transmission machine is under operation but the belt does not work. Tobacco slices cannot be blended and the precision is low.

Electrical equipment

Electrical machine broken:

The electrical machine does not work. Tobacco slices cannot be blended and the precision is low. Frequency broken: 
The frequency converter machine does not work. Tobacco slices cannot be blended and the precision is low.

Phototube broken or phototube not focused:

When the upper level phototube is broken, the dip stool will make a mistake that the material is enough and the backup equipment will not start. Tobacco slices cannot be blended. When the lower level phototube is broken, the dip stool will make a mistake that the tube has material and the backup equipment will not start. Tobacco slices cannot be blended and the precision is low.

The decoder cannot work precisely:

The decoder cannot work stably or count wrongly and precision is low.

From the above we can see that the reasons of the problem are burst equipment failure. These failures can be reduced through equipment maintenance and repair but they cannot be avoided. In a word, the reasonable design and automatic alarm are the keys.

\section{Solutions}

The features of the tobacco blending scale are:

The leaf scale is the flow control scale which operates independently under the guidance of center console. The peduncle scale, slice scale, recycled tobacco scale and bulking scale and the leaf scale are the proportion control scale, which proportion with the requirements of the instant flow of the leaf scale. The proportion control scale $=$ the instant flow of the leaf scale $*$ the setting proportion. When the proportion scale has something wrong, the actual instant flow of all the blending scales will be lower than the theoretical instant flow, and the instant blending accuracy will be low so that the quality of the product will not excellent.

The data collection of all the scale will be through plc. The communication and exchange of data will be through Ethernet.

With the features mentioned above, we designed an automatic alarm system, showing below:

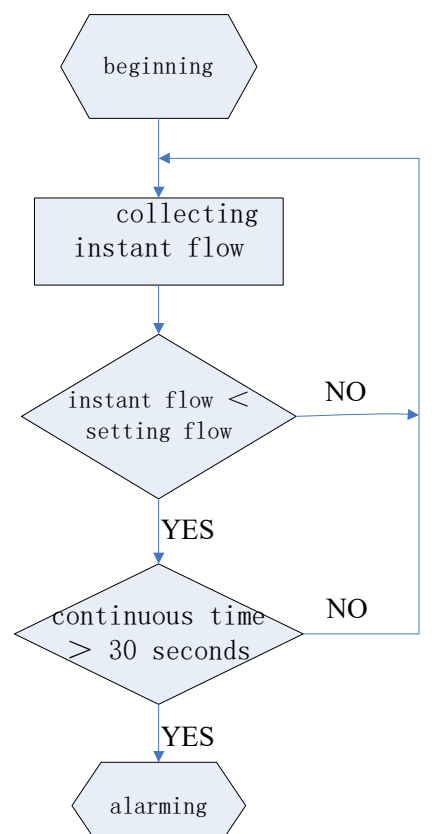

Fig.3 Flow Diagram 
According to the flow diagram, we improve the PLC procedure:

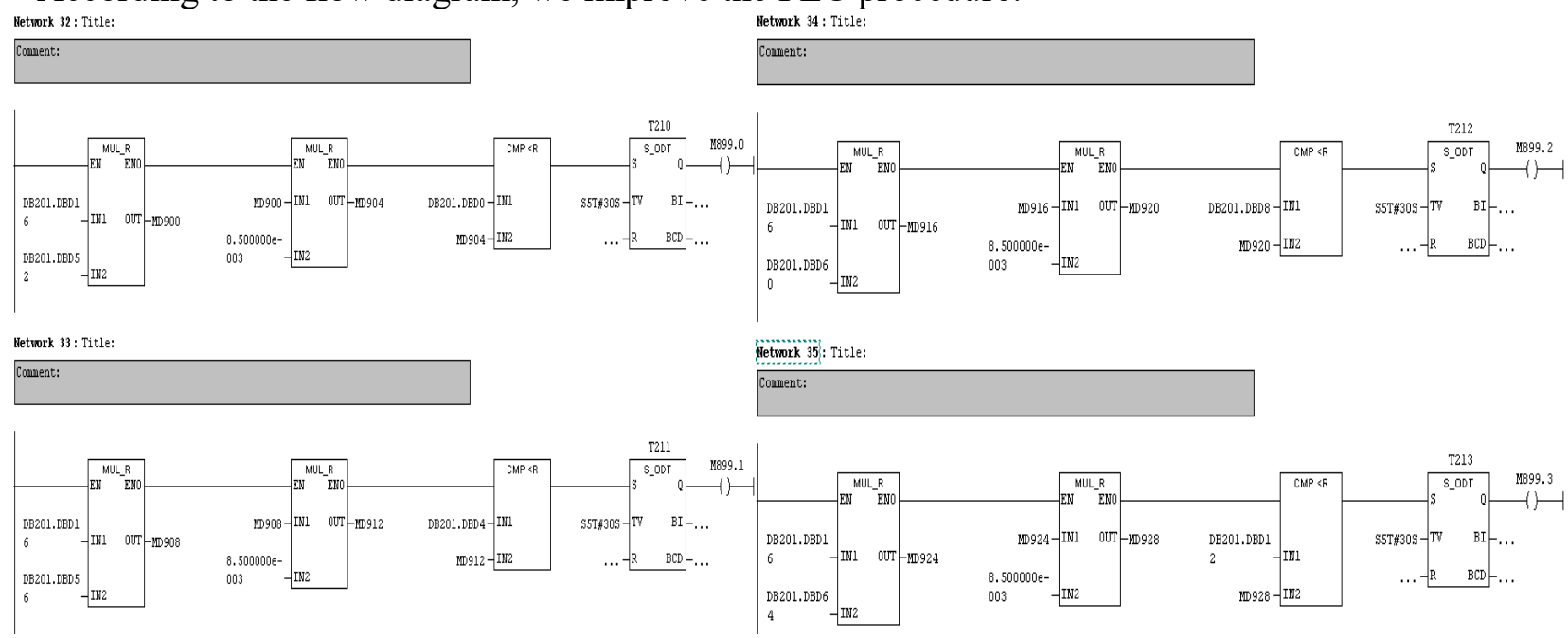

Fig.4 The PLC procedure

Variable declaration db201.dbd52

Setting proportion of peduncle blending db201.dbd0

the actual flow of the peduncle scale M899.0

Alarming mark of the peduncle scale db201.dbd56

Setting proportion of slice blending db201.dbd4

The actual flow of the slice scale M899.1

Alarming mark of the slice scale db201.dbd60

Setting proportion of expansion scale db201.dbd8

The actual flow of the expansion scale M899.2

Alarming mark of the expansion scale db201.dbd64

Setting proportion of fragment scale db201.dbd12

The actual flow of the fragment scale M899.3

Alarming mark of the fragment scale dbd201.dbd16

\section{The actual flow of the leaf scale}

Explanation of the procedure:

The leaf scale flow multiplies by the setting proportion of the peduncle scale. The result is MD900. Then multiply it by $85 \%$ and get the result of the theoretical flow MD904. Compare it with the actual peduncle flow db201.dbd0. If db201.dbd0 is less than MD904, then start the timer T210. If the accumulated time T210 is over 30 seconds, the warning sign M899.0 for the peduncle scale will appear.

The leaf scale flow multiplies by the setting proportion of the slice scale. The result is MD908. Then multiply it by $85 \%$ and get the result of the theoretical flow MD912. Compare it with the actual slice flow db201.dbd4. If db201.dbd4 is less than MD912, then start the timer T211. If the accumulated time T211 is over 30 seconds, the warning sign M899.1 for the peduncle scale will appear.

With the same process, the warnings M899.2 for bulking scale and M899.3 for blending scale will also appear.

Multiply the setting proportion of the proportion scale with the actual flow of the leaf scale, and then multiply the result with $85 \%$ of the standard flow. Compare the final result with the actual flow of the proportion scale. When the result is 30 seconds more than the actual flow, the alarming mark $\mathrm{M}$ will appear.

Meanwhile, on the monitoring screen of the central control room, 4 warning screen will be made with the monitoring software CITECT. When the instant blending accuracy is lower than 30 seconds, the warning signal $\mathrm{M}$, which has been connected with the monitoring screen of the central control room, 
will appear. The monitoring screen will show an alarming picture (see below), and the alarming horn will warning the operators to deal with the problems.

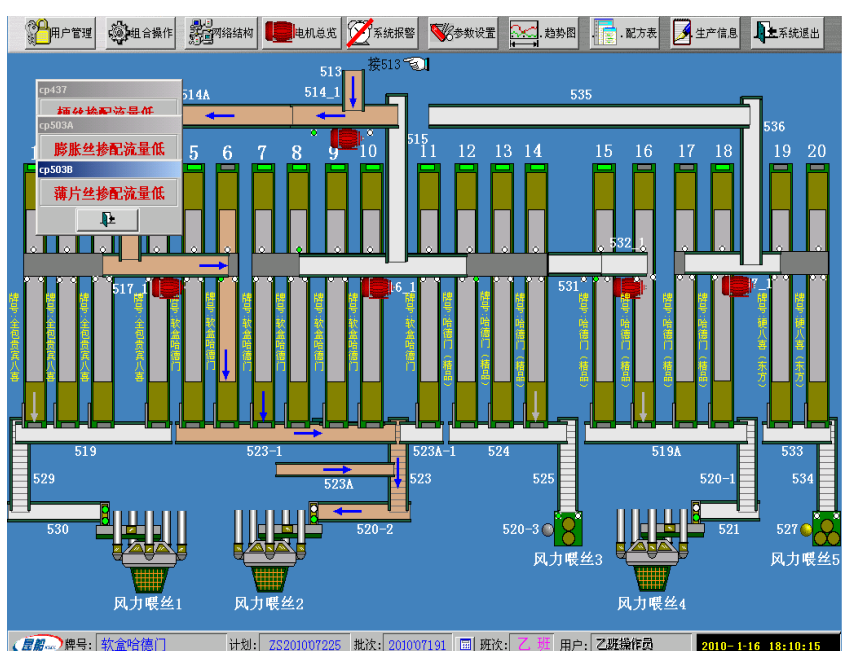

Fig.5 Example of a TWO-COLUMN figure caption

Thus, when the scales fault leads to the lowness of the instant blending accuracy, operators will find it and solve the problem. The aim of the automatic warning can be achieved and the tobacco quality will be greatly improved.

\section{Conclusion}

After the procedure operates in a normal way for a while, we will save the diagram in the tobacco product line YT607 in Qingzhou Cigarette Company and make a backup in the memory card in PLC_S400. With the reform mentioned above, the automatic alarming system is available and we can greatly improve the producing and automatic level. This is worth extending in the tobacco industry.

\section{Other Information}

This paper belongs to the University Science and Technology Plan Project of Shandong Province, China. It's number is J15LB63.

\section{References}

[1] Li Dao-lin.Electric control and PLC principle and application(SIMATIC STEP7 V5.3) [M].Beijing: Publishing House of Electronics Industry,2004.

[2] Liao Chang-chu. PLC Programming and application [M]. Beijing: Machinery industry press,2006.

[3] Wang Hao. Machine electric control and PLC[M]. Beijing: Machinery industry press,2010.

[4] Zhou Hai-tao,Bian Juan-ge. PLC For the Centralized Control of Belt Conveyor Design[J]. Electrical Application,2008.7 80-83

[5] Zhu Xue-jun,Wan Xiao-yu. Half fine boring Special Boring Machine Control System of Design Based on PLC[J]. New Technology and New Process,2009.3.26-29 
[6] Feng Xin-jun,Xiao Bing.PLC Technique Discussion and Future Development[J]. Electrical Application,2009.11 72-75

[7] Man Xiao-yu,Huang yong. Application of Intelligent Control Based On PLC in Boring Machine Transformation[J]. Industrial control computer,2007.4.56-59

[8] Feng Jing,Wang Li-li,Reconstruction of Z3040 Radial Drilling Machine Control System Based on PLC[J]. Casting technology,2008.5,45-48 